\title{
Develando econométricamente los impactos sobre la concentración atmosférica de material particulado de un proyecto de remodelación del transporte urbano: El caso del Transantiago en Chile*
} Estimating the impacts of a public transit reform on particulate matter concentration levels: the case of Transantiago in Chile

\author{
Eugenio FigueroA**, \\ ANDRÉS GóMEZ-LOBO**, \\ PABLO JORQUERA**, \\ FELIPE LABRÍN**
}

\begin{abstract}
Resumen
Estimar los impactos específicos de una reforma integral del sistema de transporte público de una gran ciudad es una tarea compleja, debido a las numerosas variables confundentes que usualmente enmascaran los efectos específicos. Este trabajo estima el impacto que la reforma integral del sistema de transporte público en Santiago de Chile, denominada Transantiago, tuvo sobre un aspecto específico: la contaminación atmosférica por material particulado (MP10) en la ciudad. Utilizando un modelo de regresión con datos diarios de panel de las distintas estaciones de monitoreo de la calidad del aire, se concluye que el Transantiago disminuyó en al menos 3,9 $\mu \mathrm{g} / \mathrm{m}^{3}$ el promedio diario de concentraciones de MP10 en la ciudad. Este efecto específico sobre la contaminación representa un ahorro estimado en costos de salud de US\$200 millones al año en el escenario medio, $12 \%$ de los cuales corresponde al ahorro de gastos en atenciones públicas. Si se excluye de la estimación los datos del primer año de operación del sistema (cuando aún no estaba en régimen), la reducción estimada de la concentración de MP10 es aún mayor. Obviamente otros efectos del Transantiago, tanto positivos como negativos, requerirían ser estimados, para obtener una evaluación completa de la reforma.
\end{abstract}

Palabras clave: Contaminación atmosférica, transporte público, salud pública, Chile.

Códigos JEL: H51, L92, Q53, R41, R48.

* Los autores agradecen los útiles comentarios recibidos de un árbitro anónimo y reconocen que cualquier error que permanezca es solo responsabilidad de ellos.

** Departamento de Economía; Universidad de Chile, Diagonal Paraguay 257, Oficina 1501; Santiago 833015; Chile. Fono: (56-2) 2978-3455. E-mail: efiguero@econ.uchile.cl; agomezlo@econ.uchile.cl; pjorquer@fen.uchile.cl; flabrino@fen.uchile.cl 


\begin{abstract}
Estimating the specific effects of a complete reform of the public transit system of a large city is a difficult task due to the confounding effects that will usually mask the impact of interest. In this study we estimate the impact of the complete reform of the public transport system in Santiago, Chile, named Transantiago, on a specific aspect of interest: particulate matter air pollution levels in the city. Using a regression model with daily panel data from air quality monitoring stations, we conclude that Transantiago reduced the daily average MP10 concentration levels in at least $3.9 \mu \mathrm{g} / \mathrm{m}^{3}$. This generates health benefits estimated to be close to US\$200 million a year in our medium scenario, $12 \%$ of which correspond to lower public sector health expenditure. If the data for the first year of operation of the system (when it was not yet in steady-state) are excluded the estimated reduction in MP10 levels is even higher. Other effects of Transantiago, positive as well as negative, would need to be estimated in order to obtain a comprehensive evaluation of the reform.
\end{abstract}

Key words: Atmospheric pollution, public transportation, public health, Chile.

JEL Classification: H51, L92, Q53, R41, R48.

\title{
1. INTRODUCCIÓN
}

El plan de reforma y reestructuración del sistema de transporte público de la ciudad de Santiago, Chile, conocido como Transantiago, demostró desde sus inicios, en febrero de 2007, serios problemas de diseño e implementación, siendo cuestionado por los medios de prensa, los usuarios y los no usuarios. ${ }^{1}$ Sin embargo, por propósito y diseño, el Transantiago debía generar una serie de beneficios para la ciudad al reducir algunas externalidades provocadas por el transporte; y hay alguna evidencia que así ha ocurrido. Por ejemplo, los accidentes que involucran a un bus de la locomoción colectiva de Santiago se redujeron $55,1 \%$, desde 6.366 en el año 2005 a 2.860 en el año $2011 .^{2}$

Por otra parte, la renovación de flota asociada al Transantiago -y que reemplazó una fracción importante de los 8.000 buses del sistema antiguo por buses que cumplieran con estándares Euro III o superiores- debería haber disminuido también la contaminación atmosférica por material particulado. ${ }^{3}$ Previo a la reforma, los buses eran una de las fuentes antropogénicas más importantes de emisiones de material particulado en la capital, estimándose que en el año

1 Mayores detalles de esta reforma, los problemas enfrentados y los ajustes realizados desde el año 2007 se encuentran en Gómez-Lobo (2012).

2 Estas cifras provienen de Comisión Nacional de Seguridad de Tránsito (CONASET) que a su vez obtuvo las estadísticas de Carabineros de Chile. Antes del Transantiago y considerando 8.000 buses en operación, en promedio cada bus estaba involucrado en un accidente cada 15 meses.

3 A diciembre de 2007, solo 57,6\% de los buses era Euro III o superior (Sectra 2007); tres años después, el 2010, casi $100 \%$ ya era Euro III o superior. 
2005 por sí solos representaban el 8\% de estas emisiones (Fernández, 2008). Así, era de esperar que una modernización significativa de la flota de buses y una racionalización de los recorridos originara una reducción de las emisiones de material particulado generadas por este sector.

Existe evidencia parcial que confirmaría la hipótesis anterior. El Centro Mario Molina Chile (2007) realizó un estudio comparando las emisiones a nivel de calle en dos arterias de importancia de la ciudad (Alameda y Gran Avenida) en el invierno de 2006, antes de la fase definitiva del Transantiago, y en el invierno de 2007, después de iniciada esta fase. Si bien para el período monitoreado hubo un aumento de $45 \%$ en las concentraciones de MP10 a nivel Metropolitano -presumiblemente como consecuencia del aumento en el uso del transporte privado, mayores emisiones de la industria y factores atmosféricos-, a nivel de calle se observó una disminución del $30 \%$ en el número de partículas ultrafinas (MP 2,5), que corresponden a las emisiones de motores de vehículos diésel, como también partículas condensadas y hollín. Además, utilizando modelos de contaminación, dicho estudio concluyó que el aporte del transporte público a la contaminación por óxidos de nitrógeno (NOx) a nivel de calle había disminuido en $50 \%$.

Sin embargo, el estudio anterior no explica la mayor contaminación a nivel de ciudad en el año 2007 comparado con el año 2006. Otros autores, Gallego, Montero y Salas (2011), estudiaron las mediciones de las estaciones de monitoreo y concluyen que hubo un aumento de $31 \%$ en las concentraciones de monóxido de carbono $(\mathrm{CO})$ en las horas punta en Santiago, presumiblemente atribuible al aumento en el uso de automóviles como consecuencia de los problemas asociados a la reforma. Sin embargo, estos autores no analizan el efecto sobre las concentraciones de material particulado.

Si bien en Santiago existen tres contaminantes atmosféricos que no cumplen con la norma establecida -el MP10 en invierno, el ozono $\left(\mathrm{O}_{3}\right)$ en verano y en menor medida el CO- es el material particulado el contaminante que hasta hace poco constituyó la mayor preocupación de la población, por ser, como se explica más adelante, el que la autoridad utilizó para decidir sobre las políticas públicas en Santiago. ${ }^{4}$

La dificultad para identificar el efecto del Transantiago sobre la contaminación en la capital es que al mismo tiempo de que se introdujo este cambio en el sistema de transporte de la ciudad, ocurrieron otros fenómenos que también afectaron la contaminación. La más relevante de estas variables confundentes al intentar estimar los efectos específicos del Transantiago es que, a partir del año 2007, se intensificaron los cortes al suministro de gas natural proveniente de Argentina, llegando a ser prácticamente 100\% en los años 2007, 2008 y 2009 para el consumo no residencial. Esto generó una sustitución generalizada por petróleo del gas natural que utilizaba el sector industrial, elevando la contribución de este sector en la contaminación de Santiago.

$4 \quad$ El año 2005 las concentraciones de CO estaban muy por debajo de la norma horaria, pero $13 \%$ sobre la norma promedio de 8 horas (Fernández, 2008). En comparación, el MP10 superaba la norma diaria en $22 \%$ y la anual en $32 \%$, mientras que el ozono superaba la norma promedio de 8 horas en $47 \%$. 
Consistente con lo anterior, es interesante notar que a partir de septiembre de 2009, cuando se pone en funcionamiento la planta de regasificación de gas natural licuado en Quintero -eliminando la dependencia de Argentina para el suministro de este combustible-, la industria vuelve a utilizar gas natural, y es justamente en 2010 cuando se registra el invierno con menores episodios críticos de contaminación desde 1997, según registros de la red MACAM2. ${ }^{5}$

Lo anterior podría implicar que, luego de desaparecer los efectos generados por los cortes de gas natural de Argentina, en el año 2010 finalmente se hicieron evidentes los beneficios del Transantiago en cuanto a reducción de emisiones y de niveles de contaminación. Desafortunadamente, esta conclusión es desafiada por al menos dos hechos. En primer lugar, por la presencia de otra variable confundente, ya que en el invierno de 2010 se registraron también condiciones meteorológicas muy favorables en Santiago, lo que favoreció la limpieza del aire. En segundo lugar, porque en el invierno de 2011 se evidenció un aumento en los episodios críticos de contaminación.

En definitiva, para establecer el impacto del Transantiago sobre la contaminación en la capital es necesario esclarecer (desenredar) la contribución de distintos factores, incluyendo las condiciones atmosféricas, los efectos de los cortes de gas de Argentina, el crecimiento económico y el propio Transantiago, entre otros factores. Para ello, en este trabajo se utilizan datos diarios de contaminación desde 1997 hasta 2009, información diaria de condiciones meteorológicas y atmosféricas, datos diarios de los cortes de suministro de gas de Argentina, además de otras variables, para estimar un modelo econométrico que explique los niveles de contaminación de material particulado grueso (MP10) en Santiago.

Los resultados indican que entre los años 2007 y 2010 se evidencia una disminución de al menos $3,9 \mu \mathrm{g} / \mathrm{m}^{3}$ en el promedio diario de la concentración de MP10 en la Región Metropolitana, que no es explicada por condiciones atmosféricas u otras variables controladas por el modelo, y que puede con cierta razón atribuirse al efecto del Transantiago.

Adicionalmente, utilizando funciones dosis-respuesta, se estiman los efectos más importantes en la salud de la población derivados de la reducción en las concentraciones de MP10 y se valorizan los cambios en mortalidad y morbilidad ocurridos, según los gastos evitados en salud. Se estima que la reducción en la contaminación equivale a un ahorro de $\mathrm{CH} \$ 417.756$ millones (o US\$ 790.2 millones $^{6}$ ) para el trienio 2007-2010, de los cuales CH\$ 49.385 millones (o US\$ 93,4 millones) corresponden al ahorro de gastos en atenciones públicas de salud.

Este estudio y sus resultados pueden ser de interés y utilidad en la región por al menos tres razones. Primero, aparte de Santiago, existen muchas otras ciudades con problemas de contaminación atmosférica, siendo el D.F. en

$\overline{5 \text { La red MACAM2 }}$, operada por el Ministerio de Salud, es una red de estaciones dedicadas a monitorear y medir las concentraciones de los contaminantes atmosféricos en distintos puntos de la ciudad de Santiago, y que se estableció el año 1997, extendiendo y mejorando técnicamente la anterior Red de Monitorio Automática de Contaminantes Atmosféricos (red MACAM).

6 Tipo de cambio corresponde a USD observado promedio 2007-2010 del Banco Central de Chile (US\$ $1=\mathrm{CH} \$ 528,7$ ). 
México un caso emblemático. En la literatura no existen estudios que modelen las concentraciones diarias de contaminantes atmosféricos, y expliquen cómo variables de política o factores exógenos (como las condiciones atmosféricas) afectan dichas concentraciones. En este sentido, este trabajo es pionero al modelar los determinantes de la contaminación atmosférica, particularmente con datos de alta frecuencia. ${ }^{7}$ Segundo, el aumento de la contaminación atmosférica ocurrido en muchas ciudades latinoamericanas en los últimos años y la necesidad de disminuir el uso del transporte privado en estas ciudades está impulsando, e impulsará en el futuro próximo, a las autoridades locales a realizar cambios importantes en los sistemas de transporte público (EIU, 2010). El presente trabajo entrega antecedentes sobre los posibles impactos que podría tener una reforma integral del transporte público de superficie en un aspecto específico, considerado crucial para las políticas públicas urbanas: la contaminación atmosférica. Tercero, es abundante la literatura, tanto de las ciencias naturales como sociales, referida a las dificultades de aislar y medir los efectos de medidas de política u otras variables sobre la contaminación del aire debido a la frecuente presencia de variables confundentes como las señaladas más arriba para el caso del Transantiago (ver, por ejemplo, Song et al., 2012 y Fernando et al., 2012). En este sentido, la metodología que aquí se propone y utiliza representa una contribución respecto del empleo de técnicas econométricas usuales para controlar adecuadamente por las variables confundentes propias del ámbito de la contaminación atmosférica y que pueden ser aplicables en países en desarrollo que implementan diferentes medidas de control y/o sufren shocks externos inesperados después de implementarlas.

El resto de este estudio está organizado de la siguiente manera: en la siguiente sección se describe el fenómeno de la contaminación atmosférica en Santiago, y se revisan las políticas adoptadas por la autoridad para su control. Luego, se propone y estima un modelo econométrico de la concentración de material particulado que permite inferir el efecto del plan Transantiago sobre la contaminación, aislándolo de otros factores relevantes. Luego se presenta un análisis de la robustez de las estimaciones. Finalmente, se reporta la valoración económica de la reducción ocurrida en la contaminación por efecto del Transantiago.

\section{La Contaminación en la Ciudad de Santiago}

Desde hace décadas, la ciudad de Santiago experimenta un alto grado de contaminación atmosférica por material particulado (MP), principalmente entre los meses de abril y agosto. Cada año se registra una serie de episodios de contaminación denominados "críticos", que son decretados por la autoridad

7 La mayor parte de la literatura se ha centrado en explicar en forma agregada, usando datos de corte transversal entre ciudades o países, los determinantes de la contaminación para establecer si existe o no una curva de Kuznets ambiental. Ver Gassebner, Lamla y Sturm (2011) para un ejemplo reciente. Otra vertiente común en la literatura es explicar los efectos en salud de la contaminación, o sea, las curvas de dosis-respuesta. Un ejemplo para Santiago es Sánchez, Valdés y Ostro (1998). Pero en la literatura no hemos encontrado publicación alguna que presente un modelo para explicar los niveles de contaminación atmosférica diaria para alguna ciudad. 

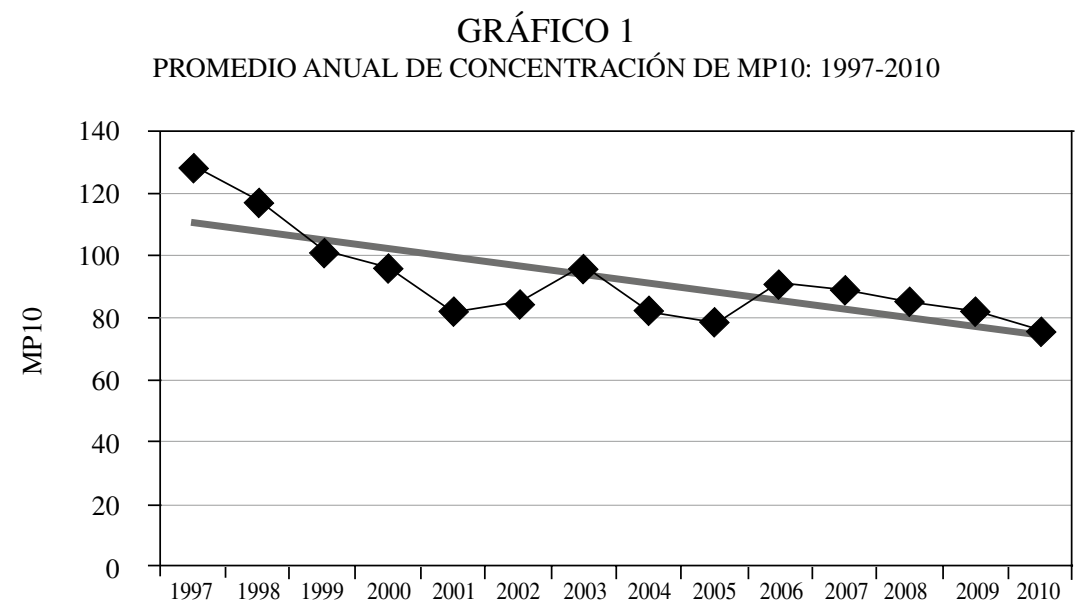

Fuente: Elaboración propia a base de datos de la Secretaría Regional Ministerial de Salud de la Región Metropolitana.

regional (Intendencia) y que se caracterizan porque la concentración de contaminantes en el aire supera los niveles máximos permitidos por los estándares de calidad ambiental de la legislación vigente, considerados como peligrosos para la salud humana. ${ }^{8}$

El Gráfico 1 presenta la evolución del promedio de concentración anual de MP10 entre los años 1997 y 2010. ${ }^{9}$ Se observa que dicho promedio muestra una tendencia decreciente, aunque moderada, indicativa de que la calidad del aire ha mejorado persistentemente en los últimos 15 años, con una mejora sustancial el año 2010. Esto se dio en un contexto de crecimiento económico, por lo que las concentraciones por unidad de producto tuvieron un descenso mayor. ${ }^{10}$

El Gráfico 2 muestra el número de episodios críticos decretados por la autoridad para la ciudad de Santiago, desglosados según su gravedad. ${ }^{11} \mathrm{Se}$

8 Los episodios críticos son clasificados como Alertas, Preemergencias o Emergencias según el nivel de las concentraciones esperadas.

9 Calculado como el promedio simple de las concentraciones horarias reportadas por la red MACAM2, para los meses críticos, entre abril y agosto; desde el año 1997 hasta el 2010; para 7 estaciones, que presentan series de datos completas para dicho período.

10 El crecimiento del PIB de la Región Metropolitana fue de 43,8\% en el período 1997-2009 (calculado a partir de las series no empalmadas de datos de PIB real para los períodos 1997-2003 (base 1996) y 2003-2009 (base 2003) del Banco Central). En el mismo período el crecimiento del PIB nacional fue de $46,7 \%$.

11 Cuando el Intendente decreta un episodio crítico, determina la gravedad del mismo (alerta, preemergencia o emergencia) de acuerdo con el nivel del Índice de Calidad del Aire referido a Partículas (ICAP), y ella define las medidas medioambientales que deben implementarse para enfrentar el episodio y proteger a la población, las que son definidas en el Plan de Prevención y Descontaminación Atmosférica (PPDA). La gravedad del episodio es mayor y las medidas que ella gatilla son más estrictas mientras mayor sea el nivel del ICAP registrado. 


\section{GRÁFICO 2 \\ NÚMERO DE EPISODIOS CRÍTICOS OBSERVADOS: 1997-2010}

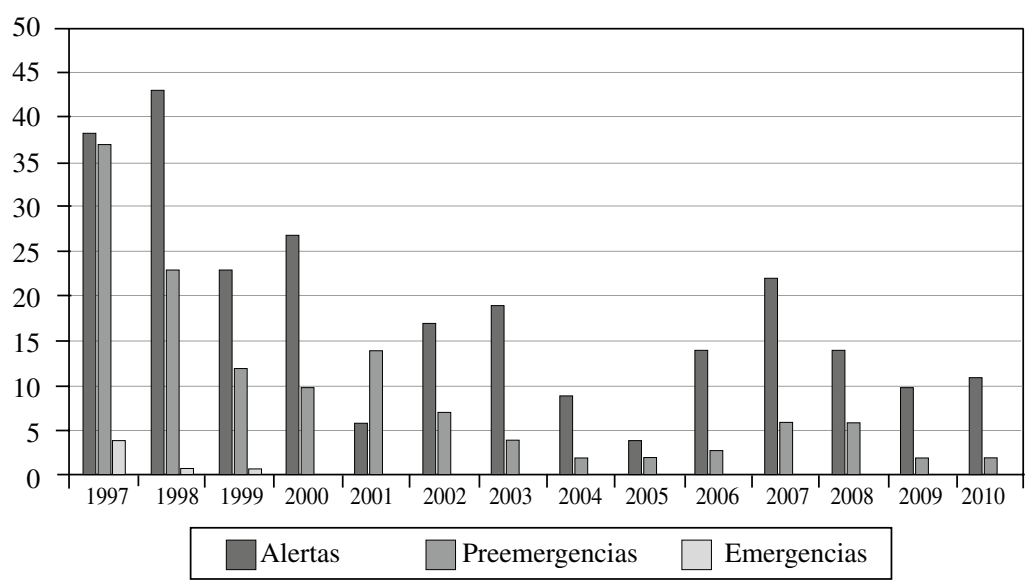

Fuente: Elaboración propia basadas en datos de Conama RM.

observa una reducción del número de episodios decretados, particularmente preemergencias y emergencias, lo que nuevamente es indicativo de las mejoras en los niveles de concentración de contaminantes.

La tendencia decreciente en la contaminación atmosférica registrada en Santiago tiene muchas y variadas causas. Primero, en el año 1998 se aprueba el Plan de Prevención y Descontaminación Atmosférica de la Región Metropolitana (PPDA) que establece medidas para la descontaminación, fijando dos ejes de acción principales: Sector transportes y combustibles, y Sector industrial, comercial y residencial (ver Merino et al., 2005).

El evidente descenso observado a partir del año 2000 se explicaría principalmente por la incorporación del gas natural al sector industrial, que reemplaza a las antiguas fuentes energéticas, como el petróleo o la leña, que son más contaminantes (CONAMA Región Metropolitana, 2009). Sin embargo, en el año 2004 comienzan a constatarse cortes del suministro de gas natural proveniente de Argentina, pudiendo cambiar la tendencia a la baja de las emisiones industriales observada hasta el año 2005. Estos cortes se hicieron más severos cada año, restringiendo la oferta de gas disponible para el sector industrial, hasta que en septiembre de 2009 se inauguró una planta de regasificación de gas natural, que permitió cubrir la demanda por este combustible mediante gas natural licuado proveniente de otros países. ${ }^{12}$ Durante el período 2004 a 2009, y en forma creciente, las emisiones del sector industrial aumentaron como consecuencia del uso de combustibles más contaminantes, asunto que se explora más adelante en el análisis empírico.

12 Los cortes del gas natural proveniente de Argentina solo afectó el consumo industrial, ya que las autoridades argentinas permitieron envíos para cubrir la demanda residencial. 
Con relación al transporte, se destaca la adopción de normas de emisión más estrictas durante el período, incluyendo medidas para la reducción de la emisión de MP10, y la introducción gradual en Santiago de un combustible diésel (denominado "Diésel Ciudad") más limpio, llegando éste, el año 2004, a tener solo 50 partes de azufre por millón. Además, durante la década se duplicó la extensión del Metro de Santiago, que al ser un medio subterráneo y eléctrico ayudaba a descongestionar y descontaminar la ciudad.

A pesar de estas medidas, en el año 2005 el sector transporte aportaba el $37,3 \%$ de las emisiones de MP10 por fuentes móviles y estacionarias, mientras que los buses aportaban el 6,7\% y la industria el 27\% (DICTUC, 2007). Es decir, a mediados de la década pasada el transporte público seguía representando una fracción significativa de las emisiones de material particulado en la ciudad. En este contexto, en febrero del año 2007 se inicia la fase final de una reforma integral del sistema de transporte público denominada Transantiago. Esta reforma incluía un conjunto de medidas, entre otras, el reordenamiento completo de las rutas, la introducción de un sistema de pago electrónico y la integración tarifaria entre los distintos servicios de buses y entre los buses y el Metro. ${ }^{13}$

En cuanto a la contaminación atmosférica, la medida más relevante del Transantiago -en el diseño original- fue la reducción del parque total de buses desde cerca de 8.000 a 4.700, aproximadamente. Además se contemplaba la renovación de parte importante de la flota de buses, que ahora debía cumplir las normas de emisión Euro III y IV. ${ }^{14} \mathrm{Si}$ bien los problemas iniciales de la reforma obligaron a aumentar la flota hasta 6.100 buses, aproximadamente, hacia el 2010 casi la totalidad de ésta la componían buses nuevos con estándar Euro III o IV.

La reforma también consideraba la construcción de vías segregadas para el transporte público, con 15 proyectos de corredores segregados con un total de $225 \mathrm{~km}$ de vías, en un horizonte de casi 20 años. ${ }^{15}$ Hacia finales de 2009, se habían completado casi 90 kilómetros de los 225 previstos en el plan. Al permitir aumentar la velocidad promedio de los buses, estas vías ayudarían a disminuir las emisiones atribuibles al transporte público. ${ }^{16}$

Por último, la reforma consideraba una reducción de los kilómetros totales recorridos por el sistema de buses. Esto se debía en parte al aumento del uso del Metro como consecuencia de la integración tarifaria y también como consecuencia de pasar de un sistema de rutas de punta a punta -que

13 Ver Gómez-Lobo (2012) para una descripción más detallada de esta reforma, su diseño original y los cambios introducidos luego de su compleja puesta en marcha.

14 Las normas de emisión EURO III y IV regulan la emisión de contaminantes para vehículos pesados y fijan los estándares de emisión de vehículos nuevos en la Unión Europea. Constituyen actualizaciones de la directiva 70/220/CCE de la Comisión Europea. La reforma también contemplaba la instalación de filtros para los buses más antiguos de la flota, pero esto nunca se llegó a materializar.

15 A octubre de 2006 se contaba con solo $16 \mathrm{~km}$ construidos.

16 Otras medidas de gestión de tránsito, como pistas solo para buses y vías exclusivas, también podrían haber contribuido a este efecto. Las primeras son vías donde existe una separación parcial y de bajo estándar entre el tráfico privado y el transporte público, de las que hay cerca de 100 kilómetros. La segunda son vías donde solo puede transitar el transporte público durante las horas punta de la semana. 
generaba una sobreposición de recorridos en las arterias más importantes de la ciudad- por un sistema de tipo troncal-alimentador. Según Beltrán y Palma (2012), los kilómetros comerciales del nuevo sistema son 30\% inferior a los del sistema antiguo.

A pesar de los tropiezos operativos iniciales, la casi completa renovación de buses al 2010, más la racionalización de los recorridos del sistema, y el uso más intensivo del Metro, hacen pensar que la implementación del Transantiago pudo haber generado un descenso en la contaminación del aire de la capital y, particularmente, de su componente MP10. Sin embargo, como se analizó antes, existen otros fenómenos de política -además de factores climatológicos- que afectaron simultáneamente con el Transantiago a la contaminación y que, por lo tanto, constituyen variables confundentes al momento de intentar aislar los efectos sobre la contaminación debidos exclusivamente a la implementación del Transantiago. Por ello, en la siguiente sección se especifica un modelo estadístico para explicar la evolución de la contaminación atmosférica por MP10 en Santiago durante el período de interés y estimar el eventual efecto del Transantiago sobre ella, una vez eliminados los efectos contemporáneos sobre la contaminación de las variables confundentes.

\section{Especificación del Modelo y Datos Empleados}

Para la modelación, la variable de interés a explicar es el nivel de contaminación atmosférica de la ciudad de Santiago, específicamente de las concentraciones diarias de MP10. Esto por dos motivos: por una parte, las concentraciones de MP10 han sido las que mayor preocupación han generado entre las autoridades $\mathrm{y}$, en concordancia con lo anterior, ellas han sido utilizadas como indicador de la calidad del aire y como variable central para el diseño y seguimiento de las políticas públicas por parte de los organismos competentes. Por otra parte, existe una disponibilidad amplia de datos sobre concentraciones de MP10 con la antigüedad suficiente para llevar a cabo un estudio estadístico.

En el presente estudio se utiliza la información oficial disponible sobre concentraciones diarias de MP10, durante el período 1997-2010, en los meses de abril a agosto (ambos inclusive) de cada año. Esto último debido a que la Comisión Nacional del Medio Ambiente (CONAMA), mediante el PPDA, propone la aplicación de medidas permanentes para el control de las emisiones de contaminantes entre el 1 de abril y el 31 de agosto de cada año. Dichos meses constituyen el período crítico para las concentraciones atmosféricas de contaminantes en la ciudad debido a las condiciones meteorológicas imperantes durante el período otoño-invierno en la cuenca de Santiago. ${ }^{17}$

Se especifica el siguiente modelo general para explicar las concentraciones diarias de MP10:

17 En estos meses se producen inversiones térmicas que reducen la ventilación de la cuenca de Santiago provocando el aumento de las concentraciones de contaminantes (Merino, 2006). 


$$
\begin{aligned}
& M P 10_{i t}=\alpha+\beta_{1} \cdot M P 10_{i t-1}+\beta_{2} \cdot \text { Misumi }_{t}+\beta_{3} \cdot \text { IMACEC }_{t}+\beta_{4} \cdot \text { Lluvias }_{t} \\
& +\beta_{5} \cdot \text { Corte } \text { gas }_{t}+\beta_{6} \cdot \text { Azufre }_{t}+\beta_{7} \cdot D_{t-1}^{\text {alerta }} \\
& +\beta_{8} \cdot D_{t-1}^{\text {preemergencia }}+\beta_{9} \cdot D_{t-1}^{\text {emergencia }}+\beta_{10} \cdot t+\gamma \cdot D_{07} \\
& +\varepsilon_{\text {día }}+\varepsilon_{\text {estación }}+\vartheta_{\text {it }}
\end{aligned}
$$

La concentración diaria de MP10 es la variable dependiente del modelo. El subíndice identifica la estación de monitoreo de calidad ambiental de la red y la fecha de la observación (días entre los meses de abril y agosto de cada año). La información proviene de la Red de Monitoreo Automática de Contaminantes Atmosféricos (red MACAM2) compuesta de ocho estaciones distribuidas en las distintas comunas del Gran Santiago (ver Figura 1). ${ }^{18}$ En el presente estudio se utilizan los datos para las siete estaciones que presentan información para todos los años del período (1997-2010), por lo que se excluyen las mediciones de la estación de la comuna de Providencia. Para transformar los datos horarios a diarios se utiliza el promedio simple de 24 horas diarias. Esta metodología es empleada en diversas investigaciones tanto internacionales como nacionales. ${ }^{19}$ En algunas especificaciones se incorpora el rezago de la variable dependiente como variable explicativa para controlar los efectos que podría tener el uso del promedio simple diario en la correlación de la contaminación entre distintos días. ${ }^{20}$

La variable Misumi es un índice de estabilidad atmosférica vertical desarrollado a fines de los años noventa por el investigador japonés Yukio Misumi junto con investigadores del Centro Nacional del Medioambiente (CENMA). Este índice mide la intensidad de la inversión térmica en la cuenca de Santiago, lo que constituye un factor crucial en la concentración de la contaminación atmosférica y se basa en la diferencia entre las temperaturas registradas en las estaciones meteorológicas operadas por el CENMA ubicadas en La Platina (a $650 \mathrm{msnm}$ ) y el cerrro Lo Prado (aproximadamente a $1.200 \mathrm{msnm}) .{ }^{21} \mathrm{El}$ índice Misumi se construye de forma horaria, por lo que en el presente trabajo se transformó a un índice diario tomando el promedio simple de las 24 horas diarias.

18 Las ocho estaciones se ubican en las comunas de Independencia, Las Condes, Santiago, Pudahuel, El Bosque, La Florida, Cerrillos, y Providencia. Además de estas estaciones, hubo algunas estaciones experimentales con mediciones ocasionales, como La Dehesa; otras se han agregado, como Cerro Navia en el 2009; y existen otras estaciones complementarias a la red MACAM2, como las de las comunas de Puente Alto, Talagante y Quilicura.

19 Ver por ejemplo Atal (2009). En una versión preliminar de este trabajo se estimó también un modelo utilizando las concentraciones a medio día (12 horas) de cada día con resultados muy similares a los reportados más adelante.

20 Esto por cuanto la concentración de las primeras horas del día estará muy relacionada con la concentración de las últimas horas del día anterior. Cuando se estimó el modelo utilizando las concentraciones a medio día (12 horas), el rezago no fue significativo.

21 La importancia de la inversión térmica en la cuenca de Santiago sobre los niveles de contaminación atmosférica de la ciudad es enfatizada en Merino (2006) y Garreaud \& Rutlant (2006). 
FIGURA 1

UBICACIÓN DE LAS ESTACIONES DE MONITOREO Y MEDICIÓN

DE LA CALIDAD DEL AIRE EN LA CIUDAD DE SANTIAGO

(Red MACAM2)

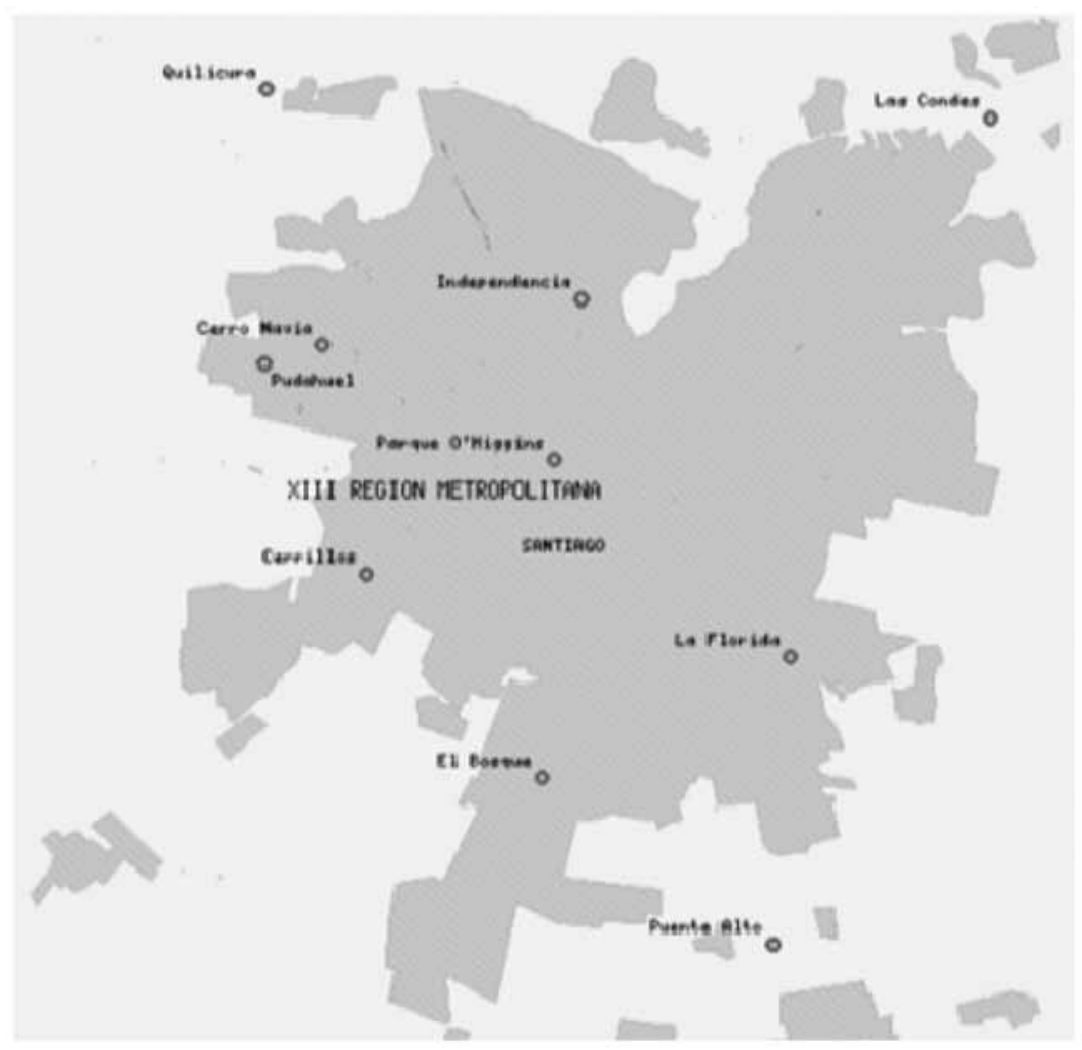

Fuente: SINIA de CONAMA.

Es esperable que mayores niveles de actividad económica generen mayores niveles de emisión de contaminantes y, por ende, todo lo demás constante, de concentración de los contaminantes (ver CONAMA, 2009). Por lo tanto, en el modelo propuesto se incluye la variable IMACEC, correspondiente al Índice Mensual de Actividad Económica (IMACEC) construido y reportado por el Banco Central de Chile. ${ }^{22}$

Las lluvias son un importante factor determinante de la concentración de MP10, porque las precipitaciones reducen su presencia en la tropósfera. La variable Lluvias se construye utilizando los datos reportados por el CENMA, que opera la estación de monitoreo meteorológica de La Platina. Debido a que esta estación no reportaba datos para los años 1999 y 2003, estos fueron

22 Los resultados no difieren si en vez de incluir este índice en forma mensual, se interpola diariamente entre los días de cada mes. 


\section{GRÁFICO 3 \\ RESTRICCIONES DIARIAS DE ENVÍOS DE GAS A LA \\ ZONA CENTRAL DE CHILE: 2004-2009}

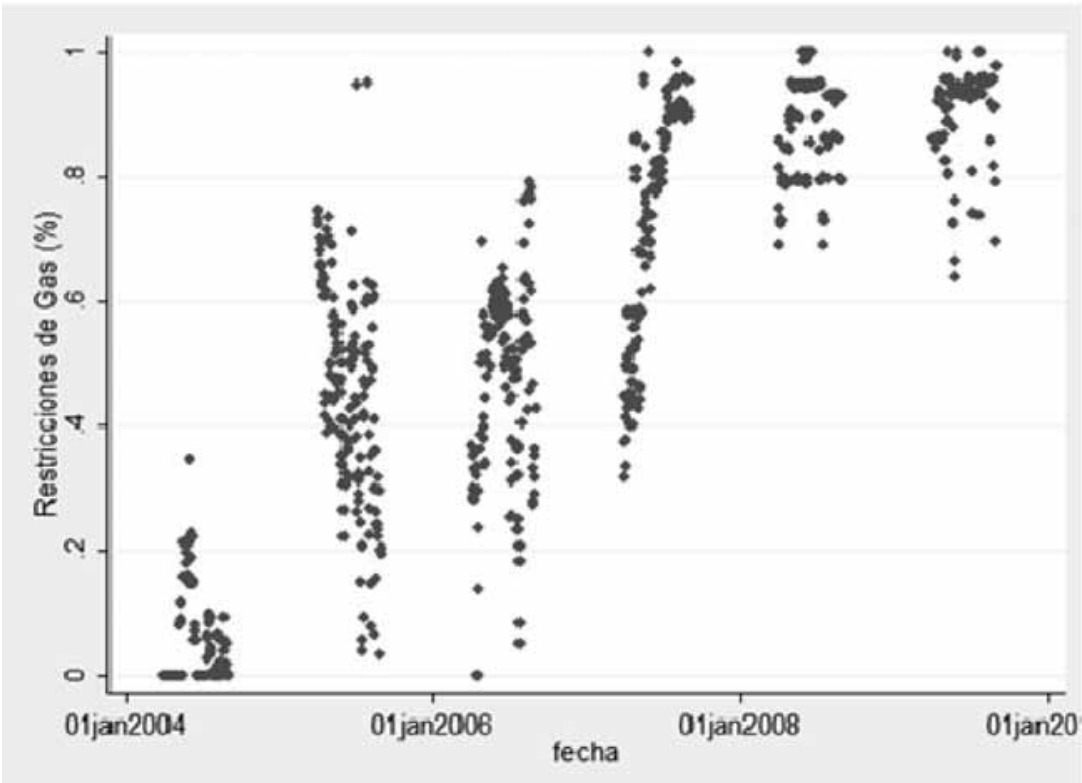

Fuente: Elaboración propia, basadas en datos de la CNE.

complementados por los datos de la Dirección Meteorológica de Chile. Esta variable está construida como una del tipo discreta que reporta la ocurrencia de un fenómeno de precipitación, sin tomar en cuenta la cantidad de horas de ocurrencia del fenómeno o milímetros de agua caída.

La variable Corte gas mide el porcentaje diario de restricción de envíos de gas natural desde Argentina para la zona central de Chile a partir del año 2004. Esta variable es relevante por cuanto habría implicado el uso de energías más contaminantes, como el petróleo, por parte del sector industrial (el sector residencial no fue afectado por los cortes). Los datos sobre las restricciones de provisión de gas son reportados, como porcentaje del total de la demanda interna, por la Comisión Nacional de Energía (CNE) y se extienden desde mayo del año 2004 hasta agosto del año 2009, fecha en que comenzó a operar la planta de recepción, almacenamiento y regasificación de Gas Natural Licuado (GNL) de Quintero. Los datos que se presentan en el Gráfico 3 muestran que en el año de entrada en vigencia del Transantiago, las restricciones de gas alcanzaron los niveles más altos en su historia previa, y continuaron creciendo hasta la entrada en operación de la planta de GNL de Quintero.

La regulación sobre la composición de los combustibles resulta relevante, ya que estos son precursores de material particulado, afectando los niveles de contaminación, principalmente de carácter secundario. Desde comienzos de la década pasada la regulación ha reducido el contenido de azufre permitido en 
los combustibles, proceso que culminó con la regulación actualmente vigente, promulgada en enero de 2004, que redujo de forma significativa la cantidad de azufre permitida en los combustibles expendidos. Por ello, el modelo incluye la variable explicativa Azufre, que corresponde a la máxima concentración de azufre permitida en los combustibles diésel para los distintos períodos, y cuyos valores se detallan en el Cuadro 1.

\section{CUADRO 1}

CONCENTRACIÓN DE AZUFRE PERMITIDA EN

COMBUSTIBLES DIÉSEL: 1997-2010

\begin{tabular}{|lc|}
\hline Año & Nivel de Azufre \\
\hline Hasta 2000 & $3.000 \mathrm{ppm}$ \\
Hasta abril de 2001 & $1.000 \mathrm{ppm}$ \\
Hasta 1 de julio de 2004 & $300 \mathrm{ppm}$ \\
Desde 2 de julio de 2004 & $50 \mathrm{ppm}$ \\
\hline
\end{tabular}

Fuente: PPDA RM y Memorias Anuales ENAP.

Es esperable que el nivel de concentraciones atmosféricas medido en un día cualquiera esté influenciado por el hecho de si el día anterior se decretó algún episodio crítico: alerta, preemergencia o emergencia. Incluir una variable que capte esta eventual relación resulta relevante, ya que al decretarse un episodio crítico la autoridad impone una serie de medidas para controlar las emisiones de fuentes fijas y móviles, lo que podría afectar la concentración de MP10 del día siguiente. Por ello, en el modelo se incluyen tres variables discretas $\left(\right.$ ' $D_{t-1}^{\text {alerta }},{ }^{\text {' }} D_{t-1}^{\text {preemergencia, }} y$ ' $D_{t-1}^{\text {emergencia }}$ ') que toman el valor de uno si en el día anterior (t-1) se decretó uno de los tipos de episodios críticos -alerta, preemergencia o emergencia ambiental- y cero en caso contrario. En la aplicación empírica del modelo, esto permite determinar no solo si existe o no el efecto esperable, sino que además la importancia relativa del efecto de cada uno de los tres tipos de episodios.

El modelo también incluye la variable $t$ de tendencia diaria, para captar los efectos del mejoramiento gradual del parque automotriz, los cambios tecnológicos, las mejoras en la productividad de los insumos, ciertos cambios en el comportamiento de los individuos, y otros fenómenos no capturados por el resto de las variables incluidas en el modelo.

El modelo incluye la variable explicativa $D 07$, que representa la motivación principal de este trabajo, pues identifica el impacto esperable sobre las concentraciones diarias de MP10 en la ciudad de Santiago que tuvo la puesta en marcha del plan Transantiago. Para ello se utiliza una variable discreta que toma el valor de uno a partir del año 2007 y que permite capturar si existe o no un cambio en el comportamiento de las concentraciones de MP10 a partir del año 2007. En la medida que no hubo cambios contemporáneos en otras variables o políticas implementadas durante el período de vigencia del Transantiago, esta 
variable debería capturar el efecto del plan sobre las concentraciones promedio de MP10. Debido a que se utiliza la variable discreta D07 señalada para identificar el efecto del Transantiago sobre la contaminación por MP10, en este trabajo se emplean varios métodos para examinar la robustez de los resultados de la estimación del modelo propuesto.

Finalmente, el modelo especifica dos tipos de efectos fijos. Primero se incluye un efecto por día de la semana, $\varepsilon_{\text {día }}$, para reflejar el hecho de que hay días con menor actividad (domingos o feriados) que los días que no son ni domingos ni feriados. Segundo, se incluye un efecto fijo por estación de monitoreo, $\varepsilon_{\text {estación }}$, para controlar por diferencias sistemáticas en las concentraciones de MP10 en las distintas zonas de la ciudad. El término $\theta_{i t}$ es un término de error que se asume bien comportado (media condicional igual a cero).

Por último, por no estar disponibles, no se incluyó en el modelo datos referidos a la intensidad del uso del parque automotriz en Santiago. Sólo están disponibles datos anuales de inscripción de vehículos, los que no capturan el uso efectivo de los vehículos, ni resultan apropiados para emplear en un modelo de carácter diario como el aquí propuesto. Por otra parte, la Unidad Operativa de Control de Tránsito (UOCT) reporta datos de flujo vehicular diario, que solo están disponibles a partir de 2004, por lo que tampoco resultan apropiados de emplear debido al período que contempla la estimación empírica del modelo aquí propuesto. La falta de información adecuada sobre la intensidad de empleo del parque vehicular puede no ser muy relevante si es correcto el juicio de algunos expertos que señalan que el efecto del transporte automotriz privado sobre las concentraciones de MP10 es bajo relativo a otras fuentes.

\section{Resultados}

Los resultados obtenidos de la estimación del modelo se presentan en el Cuadro 2. ${ }^{23}$ Las columnas (1) y (2) muestran los resultados de estimar un modelo de efectos fijos por estación (más un efecto temporal por día de la semana) no incluyendo (Modelo sin rezago) e incluyendo (1 rezago) un rezago de la variable dependiente, respectivamente. En ambos casos, los errores estándar fueron corregidos por heterscedasticidad y autocorrelación arbitraria (HAC), ya que test iniciales rechazaron la hipótesis nula de ausencia de autocorrelación de orden 1 y de homoscedasticidad. ${ }^{24}$

23 Todos los modelos incluyen variables discretas por estación de monitoreo y día de la semana que no se presentan en el Cuadro 2. Cuando se incluye la variable dependiente rezagada, el modelo corresponde a un panel dinámico y los estimadores serían sesgados en paneles cortos. Sin embargo, este sesgo disminuye a medida que aumenta $t$ y estudios de Monte Carlo como los de Judson y Owen (1999) sugieren que cuando $t>30$ ya no hay un sesgo importante de estimar el modelo mediante técnicas tradicionales de panel. En esta aplicación $t$ supera ampliamente este límite, por lo que un eventual sesgo no es relevante en este caso.

24 Se aplicó el test de Woodridge de autocorrelación de orden uno en paneles y el test de Wald de homoscedasticidad para paneles. También se probó una estimación mediante boostrap de los errores estándar con resultados muy parecidos a los reportados. 
Las columnas (3) y (4) reportan estimaciones alternativas (mediante el método Prais-Winsten) de los dos mismos modelos anteriores, pero asumiendo autocorrelación de orden uno, y errores estándar robustos a heteroscedasticidad arbitraria entre paneles y correlación contemporánea de los errores entre los distintos paneles.

Estimaciones preliminares indicaron que la variable de episodios de emergencia en el día previo no resultó significativa en ninguna de las regresiones y se eliminó de los modelos presentados en el Cuadro 2. Esto se puede explicar por la falta de información estadística, debido a que estos episodios son poco frecuentes, y han ido disminuyendo durante la última década. La variable rezagada de lluvias tampoco resultó significativa estadísticamente y se omitió. Lo anterior indica que el efecto de las lluvias no se prolonga en el tiempo, o bien, la naturaleza discreta de esta variable no permite hacer este tipo de inferencias, debido a que solo indica si hubo precipitaciones, y no toma en cuenta la intensidad de las mismas o su duración.

Del Cuadro 2 se observa que en general todas las variables tienen el signo esperado y son altamente significativas, particularmente en los modelos con la variable dependiente rezagada. ${ }^{25}$

Los resultados para el índice Misumi confirman la relación positiva entre las condiciones de la inversión térmica de la cuenca y los niveles de concentración de MP10. Esto ratifica el condicionamiento climático de la contaminación atmosférica en la cuenca de Santiago. Esta relación es decreciente, como se observa del signo negativo relacionado con la variable Misumi al cuadrado $\left(\right.$ Misumi $\left.^{\wedge 2}\right){ }^{26}$

La actividad económica también tiene un impacto positivo sobre las concentraciones. La variable IMACEC es mensual, pero los resultados reportados no se alteran significativamente si se utiliza esta variable interpolada diariamente.

Tal como se esperaba, se observa que restricciones mayores en los envíos de gas desde Argentina impactan de forma directa la contaminación diaria por MP10, donde $100 \%$ de restricción al suministro de gas en un día implica un aumento de más de $15 \mu \mathrm{g} / \mathrm{m}^{3}$ en las concentraciones promedio diarias de MP10. ${ }^{27}$ Esto se explicaría por una reconversión del sector industrial hacia fuentes energéticas más contaminantes para hacer frente a los cortes en los suministros. ${ }^{28}$

Por otra parte, se observa un efecto negativo de las lluvias sobre las concentraciones de MP10 en un mismo día, lo que era de esperarse debido al efecto precipitador del material particulado en suspensión de las lluvias. Como se señaló

25 Como se explicó anteriormente, la inclusión del rezago tiene sentido, ya que la variable de concentración es un promedio simple de los datos horarios, por lo que las concentraciones de la noche anterior están correlacionadas con las primeras horas del día siguiente. Si se estima el modelo con las concentraciones a medio día, en lugar del promedio diario, los rezagos ya no son significativos.

26 Estimar el modelo sin incluir la variable Misumi al cuadrado no altera los resultados presentados aquí.

27 En el caso del modelo con rezagos, el impacto de largo plazo es similar. Para obtener el impacto de largo plazo se debe dividir el parámetro asociado a los cortes de gas por uno menos la suma del coeficiente de la variable dependiente rezagada.

28 La mayor parte de las calderas y equipos del sector industrial son duales, en el sentido de que permiten el uso de varios combustibles. Esta conversión se puede hacer en forma diaria. 


\section{CUADRO 2}

RESULTADOS DE LA ESTIMACIÓN DEL MODELO DE CONCENTRACIONES DIARIAS DE MP10 EN LA CIUDAD DE SANTIAGO, 1997-2010

\begin{tabular}{|c|c|c|c|c|}
\hline Variables & $\begin{array}{c}(1) \\
\text { Modelo } \\
\text { sin rezago }\end{array}$ & $\begin{array}{c}(2) \\
1 \\
\text { rezago }\end{array}$ & $\begin{array}{c}(3) \\
\text { Modelo } \\
\text { sin rezago }\end{array}$ & $\begin{array}{c}(4) \\
1 \\
\text { rezago }\end{array}$ \\
\hline Método estimación & $\begin{array}{l}\text { Efectos fijos } \\
\text { con VC } \\
\text { robusta HAC }\end{array}$ & $\begin{array}{l}\text { Efectos fijos } \\
\text { con VC } \\
\text { robusta HAC }\end{array}$ & $\begin{array}{l}\text { Prais-Winsten } \\
\text { con VC } \\
\text { robusta }\end{array}$ & $\begin{array}{c}\text { Prais-Winsten } \\
\text { con VC } \\
\text { robusta }\end{array}$ \\
\hline 1 rezago MP10 & & $\begin{array}{l}0,328^{* * *} \\
(0,008)\end{array}$ & & $\begin{array}{l}0,252 * * * \\
(0,007)\end{array}$ \\
\hline Misumi & $\begin{array}{l}6,508 * * * \\
(0,130)\end{array}$ & $\begin{array}{l}5,211^{* * * *} \\
(0,118)\end{array}$ & $\begin{array}{l}5,485^{* * * *} \\
(0,106)\end{array}$ & $\begin{array}{l}5,380 * * * \\
(0,105)\end{array}$ \\
\hline Misumi $^{\wedge 2}$ & $\begin{array}{l}-0,104 * * * \\
(0,004)\end{array}$ & $\begin{array}{l}-0,085^{* * *} \\
(0,004)\end{array}$ & $\begin{array}{l}-0,079 * * * \\
(0,003)\end{array}$ & $\begin{array}{l}-0,085^{* * *} \\
(0,003)\end{array}$ \\
\hline IMACEC & $\begin{array}{l}1,532 * * * \\
(0,079)\end{array}$ & $\begin{array}{l}1,051 * * * \\
(0,067)\end{array}$ & $\begin{array}{l}1,549 * * * \\
(0,102)\end{array}$ & $\begin{array}{l}1,074 * * * \\
(0,073)\end{array}$ \\
\hline Gas & $\begin{array}{l}15,53^{* * * *} \\
(2,164)\end{array}$ & $\begin{array}{l}10,54 * * * \\
(1,794)\end{array}$ & $\begin{array}{l}15,30 * * * \\
(2,879)\end{array}$ & $\begin{array}{l}11,64 * * * \\
(2,183)\end{array}$ \\
\hline Lluvia & $\begin{array}{l}-11,05^{* * * *} \\
(0,730)\end{array}$ & $\begin{array}{l}-10,31 * * * * \\
(0,654)\end{array}$ & $\begin{array}{l}-9,970 * * * \\
(0,607)\end{array}$ & $\begin{array}{l}-10,56^{* * * *} \\
(0,607)\end{array}$ \\
\hline Tendencia & $\begin{array}{l}-0,029 * * * \\
(0,001)\end{array}$ & $\begin{array}{l}-0,021 * * * * \\
(0,001)\end{array}$ & $\begin{array}{l}-0,029^{* * *} \\
(0,001)\end{array}$ & $\begin{array}{c}-0,023^{* *} \\
(0,001)\end{array}$ \\
\hline Alerta $_{\mathrm{t}-1}$ & $\begin{array}{l}5,373^{* * *} \\
(0,876)\end{array}$ & $\begin{array}{l}-2,625^{* * *} \\
(0,837)\end{array}$ & $\begin{array}{l}1,868^{* *} \\
(0,744)\end{array}$ & $\begin{array}{c}-0,890 \\
(0,759)\end{array}$ \\
\hline Preemergencia $_{t-1}$ & $\begin{array}{l}3.107^{*} \\
(1.735)\end{array}$ & $\begin{array}{l}-4,629^{* *} \\
(1,539)\end{array}$ & $\begin{array}{c}-0,229 \\
(1,174)\end{array}$ & $\begin{array}{l}-2,517 * * \\
(1,199)\end{array}$ \\
\hline Azufre & $\begin{array}{l}0,002^{* * *} \\
(0,001)\end{array}$ & $\begin{array}{c}0,000 \\
(0,001)\end{array}$ & $\begin{array}{l}0,002^{* * * *} \\
(0,001)\end{array}$ & $\begin{array}{c}0,001 \\
(0,001)\end{array}$ \\
\hline D07 & $\begin{array}{l}-5,142^{* * * *} \\
(1,365)\end{array}$ & $\begin{array}{c}-2,610^{* *} \\
(1,121)\end{array}$ & $\begin{array}{l}-5,392 * * * \\
(1,804)\end{array}$ & $\begin{array}{l}-3,392 * * * \\
(1,280)\end{array}$ \\
\hline Constante & $\begin{array}{l}323,9^{* * * *} \\
(14,10)\end{array}$ & $\begin{array}{l}234,4 * * * \\
(11,98)\end{array}$ & $\begin{array}{l}320,2^{* * * *} \\
(17,69)\end{array}$ & $\begin{array}{l}253,1^{* * * *} \\
(12,81)\end{array}$ \\
\hline Observaciones & 13.346 & 13.247 & 13.346 & 13.247 \\
\hline $\mathrm{R}^{2}$ & 0,59 & 0,65 & 0,50 & 0,65 \\
\hline $\mathrm{F}$ Test $(\mathrm{P}>\mathrm{F})$ & 0,000 & 0,000 & 0,000 & 0,000 \\
\hline Efecto LP & $-5,142$ & $-3,884$ & $-5,392$ & $-4,535$ \\
\hline
\end{tabular}

Fuente: Elaboración propia.

Nota: Errores estándar entre paréntesis $* * * \mathrm{p}<0,01, * * \mathrm{p}<0,05,{ }^{*} \mathrm{p}<0,1$. Los errores estándar de los modelos de la columna (1) y (2) son corregidos por autocorrelación y heteroscedasticidad arbitraria usando un kernel de Bartlett con ancho de banda igual a 2. Los modelos (3) y (4) fueron estimados mediante regresiones Prais-Winsten asumiendo autocorrelación de orden 1 específico a cada panel y corrigiendo los errores estándar por heteroscedasticidad arbitraria en cada panel y correlación contemporánea en los errores entre paneles. 
anteriormente, este efecto de abatimiento del MP10 de las precipitaciones no se extiende temporalmente más allá del día en que ocurre la lluvia.

La variable Tendencia revela una propensión en el tiempo a la baja de los niveles de contaminación por MP10, lo que podría estar relacionado con una mejora tecnológica constante del parque automotriz en materia medioambiental (mayor eficiencia, por ejemplo) u otros cambios tecnológicos graduales.

Es particularmente interesante observar el resultado de las variables discretas para las alertas y preemergencias ocurridas el día anterior. Los resultados de la columna (1) indican que, para cualquiera día, un episodio de alerta o preemergencia ambiental decretado el día anterior está asociado a mayores concentraciones de MP10 para ese día. Sin embargo, al incluir la variable dependiente rezagada (columna (2)) este efecto se torna negativo, como era de esperarse. Además, el impacto negativo es menor en el caso de las alertas que las preemergencias, lo que es razonable si se considera que estos últimos episodios gatillan medidas regulatorias más agresivas, como el cierre de fuentes fijas de emisión y una mayor restricción a los vehículos que pueden circular ese día. En los modelos (3) y (4) los resultados son menos claros, pero cualitativamente similares a lo descrito para los primeros dos modelos.

Respecto del azufre, se observa que existe una relación positiva entre los contenidos del mismo en los combustibles expendidos y las concentraciones evidenciadas de MP10 en la ciudad. Sin embargo, este efecto no es estadísticamente significativo en los modelos con rezago de la variable dependiente. Esto último podría deberse en parte a que la variable de tendencia podría estar capturando parte del efecto de la disminución del contenido de azufre en los combustibles, ya que como se muestra en el Cuadro 1, la reducción de este contaminante fue gradual durante la década pasada.

Aunque no se presentan en el Cuadro, las variables de efectos fijos por estación de monitoreo indican que las concentraciones de MP10 recogidas en la estación de Las Condes son considerablemente menores a las otras estaciones. Esto puede deberse a que el uso del suelo en dicha comuna y sus aledañas es antes que todo residencial (y no industrial) contando, además, con más áreas verdes que otras comunas, y por otro lado, a que las mejores condiciones económicas de sus habitantes permiten un uso de superiores y más modernos vehículos y equipos de calefacción. Por otra parte, las estaciones de La Florida y Pudahuel, corresponden a las dos estaciones con un mayor registro de concentraciones de MP10, debido a sus características geográficas y de ventilación.

Tampoco se reportan los efectos fijos del día de la semana. Pero los resultados para estas variables son consistentes con lo esperado. El día viernes corresponde al de mayor nivel de concentraciones en la semana. Tal situación se entiende debido a un aumento de los viajes realizados hacia fuera de la ciudad, y un efecto acumulativo de la actividad de la semana laboral. Se destaca también que en los días domingo hay una menor concentración, debido a una menor actividad generadora de contaminantes, y este efecto persiste hasta el día lunes.

Finalmente, se observa que, consistente con la hipótesis central de este estudio, las concentraciones de MP10 han disminuido desde el año 2007 en adelante. En el modelo (1) sin rezago esta disminución es de $-5,142 \mu \mathrm{g} / \mathrm{m}^{3}$, mientras que con rezago disminuye a $-2,61 \mu \mathrm{g} / \mathrm{m}^{3}$ (modelo columna (2)) en el corto plazo, lo que corresponde a una disminución de $-3,9 \mu \mathrm{g} / \mathrm{m}^{3}$ en el largo plazo (ver última fila del Cuadro 2 para los efectos de largo plazo). Los efectos en los modelos 
(3) y (4) son algo más altos $\left(-5.4 \mu \mathrm{g} / \mathrm{m}^{3}\right.$ y $-4,5 \mu \mathrm{g} / \mathrm{m}^{3}$, respectivamente). Por lo tanto, esta evidencia empírica es conclusiva para rechazar la hipótesis de que la implementación, a partir del año 2007, del plan Transantiago de modernización del sistema de transporte urbano en Santiago no provocó una disminución en la contaminación por MP10 en dicha ciudad.

\section{Robustez de La Estimación ${ }^{29}$}

La inferencia respecto del impacto del Transantiago se hace utilizando una variable discreta para el período 2007 a 2010. Esta inferencia podría ser errada si dicha variable estuviese captando no linealidades o efectos de tendencia no controlados en el modelo. Para examinar la robustez de los resultados se realizaron dos ejercicios adicionales. Primero, se estimó el modelo (2) del Cuadro 2 incorporando una tendencia cuadrática para analizar si existen no linealidades en la especificación. Segundo, se realizó un test de falsificación, incorporando una variable dicotómica en los años 2005 y 2006, para luego realizar la misma regresión especificada anteriormente pero solo hasta el año 2006 (previo a la implementación del Transantiago). Si esta variable resulta ser estadísticamente significativa y de signo negativo, podría ser entonces que la variable D07 en los modelos anteriores, estuviese capturando alguna no linealidad o variables omitidas que nada tienen que ver con el Transantiago.

Los resultados para las variables de interés se presentan en el Cuadro 3. Se puede ver que al incluir una tendencia al cuadrado, esta variable no resulta estadísticamente significativa y además no cambia el signo ni la significancia de la variable D07. Por otro lado, al acortar la muestra hasta el año 2006 e incluir una variable discreta para el año 2005 y 2006, esta última variable no demuestra ser estadísticamente significativa. Por lo tanto, ambos resultados sugieren que los resultados expuestos en la sección anterior son robustos y que la variable D07 está efectivamente controlando por algo que ocurrió a partir de esa fecha.

Por último, es posible que la variable D07 estuviese capturando en parte las falencias del sistema en sus inicios donde hubo una flota muy reducida de buses operando que no refleja las emisiones del sistema en estado de régimen. Para ello se estimó el modelo eliminando los datos del año 2007 (pero sí incluye del 2008 al 2010). Los resultados se presentan en la columna (3) del Cuadro 3, donde se evidencia que la variable discreta $D 07$ sigue siendo negativa y altamente significativa. Además, el valor absoluto del coeficiente es mayor, por lo que se concluye que la reducción de la contaminación fue mayor entre el 2008 y 2010. Esto es consistente con el hecho de que una parte importante de la flota se renovó a finales del 2007 (los buses de la Troncal 5) y hacia el 2010 prácticamente toda la flota había sido renovada por buses Euro III, Euro IV o superiores. ${ }^{30}$

\footnotetext{
29 Agradecemos a Eduardo Fajnzylber por proponer varios de los test de robustez que se presentan aquí.

30 Las Figuras $\mathrm{N}^{\circ} 1 \mathrm{~b}, \mathrm{~N}^{\circ} 2$ a y No 3 de Beltrán y Palma (2012) indican que el número de buses, los kilómetros de servicio y los kilómetros comerciales del sistema (lo más relevante para determinar emisiones) fueron bastante estables a partir del año 2008, por lo que tampoco debería haber un sesgo importante por estos factores.
} 
Todo los tests comentados permiten concluir que las estimaciones del modelo econométrico son robustas.

\author{
CUADRO 3 \\ ANÁLISIS DE ROBUSTEZ \\ (Modelo con rezago)
}

\begin{tabular}{|c|c|c|c|}
\hline Variables & $\begin{array}{l}\text { (1) } \\
\text { Modelo tendencia } \\
\text { cuadrática }\end{array}$ & $\begin{array}{l}\text { (2) } \\
\text { Modelo datos } \\
\text { hasta } 2006\end{array}$ & $\begin{array}{c}\text { (3) } \\
\text { Modelo sin datos } \\
\text { del año } 2007\end{array}$ \\
\hline Tiempo & $\begin{array}{c}-0,028 * * \\
(0,012)\end{array}$ & $\begin{array}{l}-0,024 * * * \\
(0,001)\end{array}$ & $-0,019 * * *$ \\
\hline tiempo 2 & $\begin{array}{c}0,000 \\
(0,000)\end{array}$ & - & - \\
\hline $\mathrm{d} 07$ & $\begin{array}{c}-3,019 * * \\
(1,361)\end{array}$ & - & $\begin{array}{c}-7,055 * * * \\
(1,269)\end{array}$ \\
\hline d05 & - & $\begin{array}{c}2,44 \\
(1,693)\end{array}$ & - \\
\hline Observaciones & 13.247 & 10.090 & 12.190 \\
\hline R2 & 0,65 & 0,66 & 0,65 \\
\hline $\mathrm{F}$ test $(\mathrm{P}>\mathrm{F})$ & 0,000 & 0,000 & 0,000 \\
\hline
\end{tabular}

Fuente: Elaboración propia.

Nota: Los errores estándar son corregidos por autocorrelación y heteroscedasticidad arbitraria usando un kernel de Bartlett con ancho de banda igual a 2. Errores estándar entre paréntesis. $* * * \mathrm{p}<0,01, * * \mathrm{p}<0,05, * \mathrm{p}<0,1$. No se presentan los resultados para los otros coeficientes del modelo.

\title{
6. Valoración Económica de las Disminuciones en laS CONCENTRACIONES DE MP10
}

En esta sección se valoran económicamente los efectos medioambientales que, de acuerdo con los resultados de la sección anterior, son atribuibles al Transantiago. Estos efectos se refieren de manera específica a la disminución de las concentraciones de MP10 a partir del año 2007, cuando el Transantiago entró en funcionamiento. Nótese que esta valoración tenderá a subestimar el verdadero valor de estos efectos, ya que las concentraciones de MP10 tienen múltiples repercusiones en el bienestar de los individuos expuestos a dichas concentraciones. El presente estudio solo se centra en la dimensión de los menores costos en salud provocados por la reducción de las concentraciones diarias de MP10.

Para estimar la reducción en las concentraciones de MP10 a que está expuesta la población de Santiago se utilizan los resultados del modelo de la columna (2) del Cuadro 2, por ser los más conservadores entre los modelos con rezagos. 
Como ya se señaló, dicho modelo indica que en el largo plazo el efecto en las concentraciones de MP10 es de $-3,9 \mu \mathrm{g} / \mathrm{m}^{3}$.

Se calculan los efectos de la reducción de la contaminación sobre la salud humana, específicamente en mortalidad y morbilidad (admisiones hospitalarias por enfermedades respiratorias, admisiones hospitalarias por enfermedades cardiovasculares, visitas a salas de urgencia por enfermedades respiratorias, días de actividad restringida, enfermedades respiratorias bajas en niños, bronquitis crónicas, síntomas respiratorios agudos y ataques de asma). Para ello se usan funciones de daño o concentración-respuesta lineales recopiladas por Sánchez, Valdés y Ostro (1998) y los ponderadores utilizados por dichos autores para el escenario medio. ${ }^{31}$

Para calcular los cambios en riesgos de salud de la población se utiliza la siguiente ecuación:

$$
\Delta E S_{i}=\alpha^{*} \operatorname{Pob}_{i} * \Delta M P 10_{i}
$$

donde:

$\Delta E S_{i}=$ Cambio en el riesgo salud de la población, del efecto en salud $i$.

$\alpha \quad=$ Ponderador de la función de daño, equivale a la pendiente de la misma.

$P o b_{i}=$ Población en riesgo relativo al efecto en salud $i$.

$\Delta M P 10_{i}=$ Cambio en la concentración atmosférica de $M P 10$

Como población expuesta o afectada se considera al total de población de la provincia de Santiago, ya que como se dijo anteriormente, se estimó un efecto parejo para toda la ciudad, y es en esta provincia donde operó el plan Transantiago. Estas cifras son reportadas por el Instituto Nacional de Estadísticas (INE).

En algunos casos, las funciones de daño requieren el uso de las admisiones hospitalarias en lugar de población en la ecuación anterior. En estos casos se usan los datos entregados por el Departamento de Estadísticas e Información de Salud (DEIS) del Ministerio de Salud (MINSAL) para todos los Servicios de Salud de la región, los que se encuentran desglosados únicamente para los años 2008 y 2009, y de los que se usan los correspondientes al período abril-agosto. Para el año 2007 solo están disponibles los datos de hospitalizaciones totales para la región, por lo que, para establecer las admisiones hospitalarias específicas para las enfermedades en cuestión, se supuso que la proporción de estas era igual a la proporción promedio de los años 2008 y 2009. Sólo se utilizan las admisiones hospitalarias del sector público, debido a que los registros del sector privado no se encuentran disponibles, razón por lo que se puede indicar que las estimaciones para las admisiones hospitalarias estarían subvaluadas, aun cuando se considera suficiente la información, ya que el sector público representa más del $70 \%$ de las atenciones totales.

31 Las funciones de daño o dosis-respuesta relacionan estadísticamente la exposición a contaminantes atmosféricos de una población con diferentes efectos de salud observados en la misma población, entregando como resultado un número de casos de enfermedad (morbilidad) o de vidas perdidas (mortalidad) para los distintos niveles de exposición. 


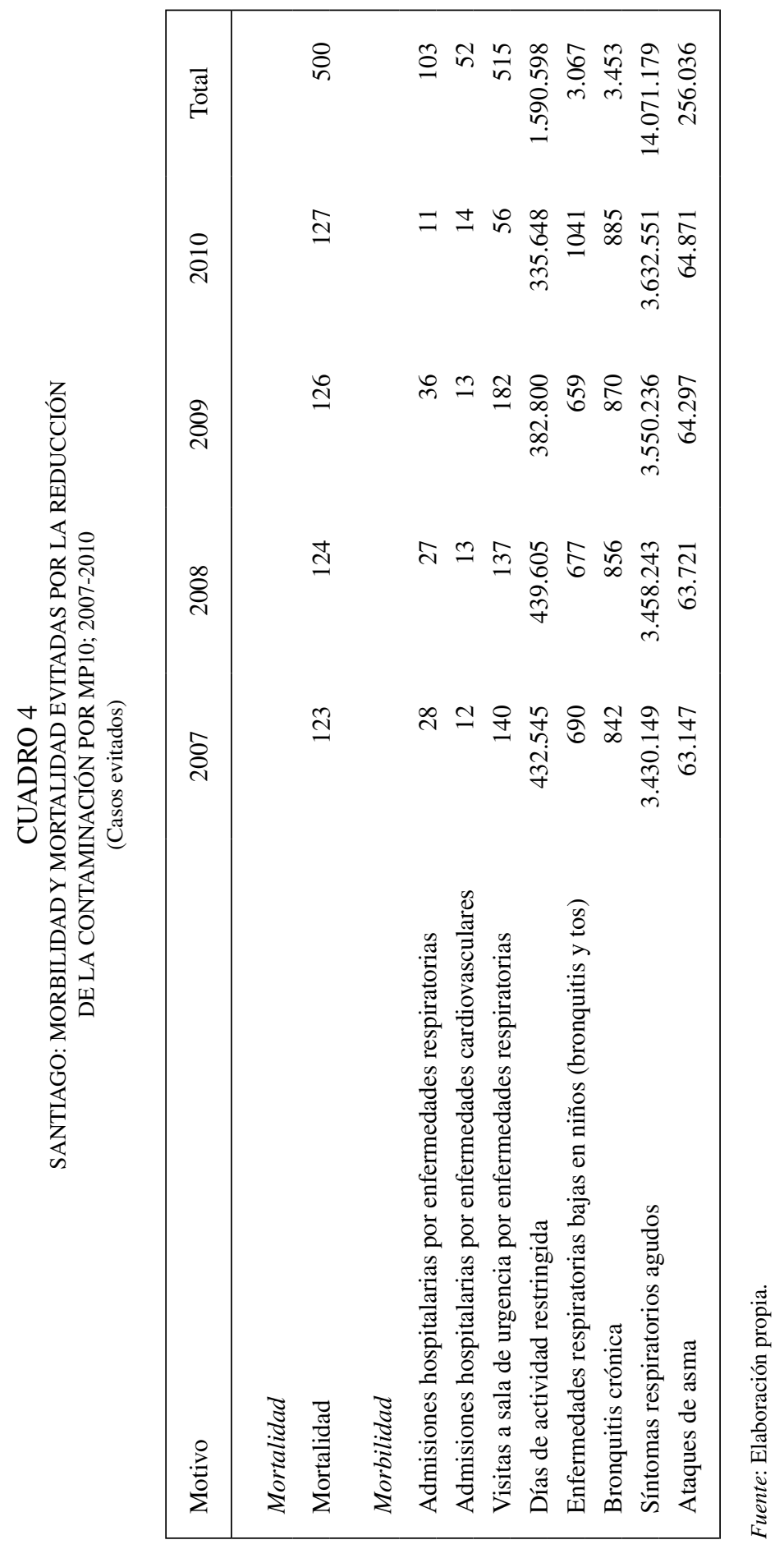


Para las enfermedades respiratorias bajas se consideraron las atenciones por neumonía (J12-J18), bronquitis y bronquiolitis aguda (J20-J21) y crisis obstructiva bronquial (J40-J46). Además se consideró la tasa de mortalidad cruda calculada por CEPAL para el quinquenio 2005-2010 de 5,36 muertes por 1.000 habitantes, debido a que aún no se cuenta con los datos oficiales de mortalidad para el año 2009.

El Cuadro 4 presenta las estimaciones de efectos para el escenario de nivel medio producto de la disminución de las concentraciones diarias promedio de MP10 en el período invernal, desde el 2007 al 2010, atribuibles al Transantiago.

Para la valoración económica de los impactos en salud se usaron los cálculos realizados por Holz (2000), los que debieron ser actualizados a precios del 2010. Este autor calcula para cada enfermedad tanto sus costos directos como indirectos. Los primeros se definen en función de los costos promedio de tratar la enfermedad. Dichos valores fueron actualizados según la variación del índice de precios al consumidor de salud reportado por el INE. Los segundos representan los días de trabajo perdidos, tanto por la enfermedad del individuo enfermo en hospitalizaciones y atenciones de urgencia como por las horas de trabajo perdidas por quienes deben acompañar a menores de edad en dichos procedimientos. Estos costos se actualizaron mediante la variación del Índice de Remuneraciones Generales (Ffrench-Davis, 2010).

Los valores finales utilizados se presentan en el Cuadro 5.

CUADRO 5

COSTOS DE CADA ENFERMEDAD

(CH\$ del 2010)

\begin{tabular}{|lr|}
\hline Motivo & \multicolumn{1}{c|}{ Valor } \\
& (Pesos de 2010) \\
\hline Mortalidad & 30.797 .105 \\
Admisión hospitalaria por enfermedades respiratorias & 978.703 \\
Admisión hospitalaria por enfermedades cardiovascular & 19.683 .872 \\
Visitas a sala de urgencias & 44.765 \\
Enfermedad baja en niños & 100.846 \\
Bronquitis crónica & 82.704 .641 \\
Síntomas respiratorios agudos & 5.308 \\
Ataques de asma & 100.293 \\
Día de actividad restringida & 9.399 \\
\hline
\end{tabular}

Fuente: Elaboración propia. Actualización de valores de Holz (2000).

Considerando todo lo anterior, para el escenario medio se estiman los ahorros por menores costos de salud que se presentan en el Cuadro 6. Estos se estiman en CH 104 mil millones en promedio entre los años 2007 y 2010. Esta cifra corresponde a un valor cercano a los US\$ 216 millones al año. ${ }^{32}$

32 Se utiliza un tipo de cambio de CLP 480 por USD. 


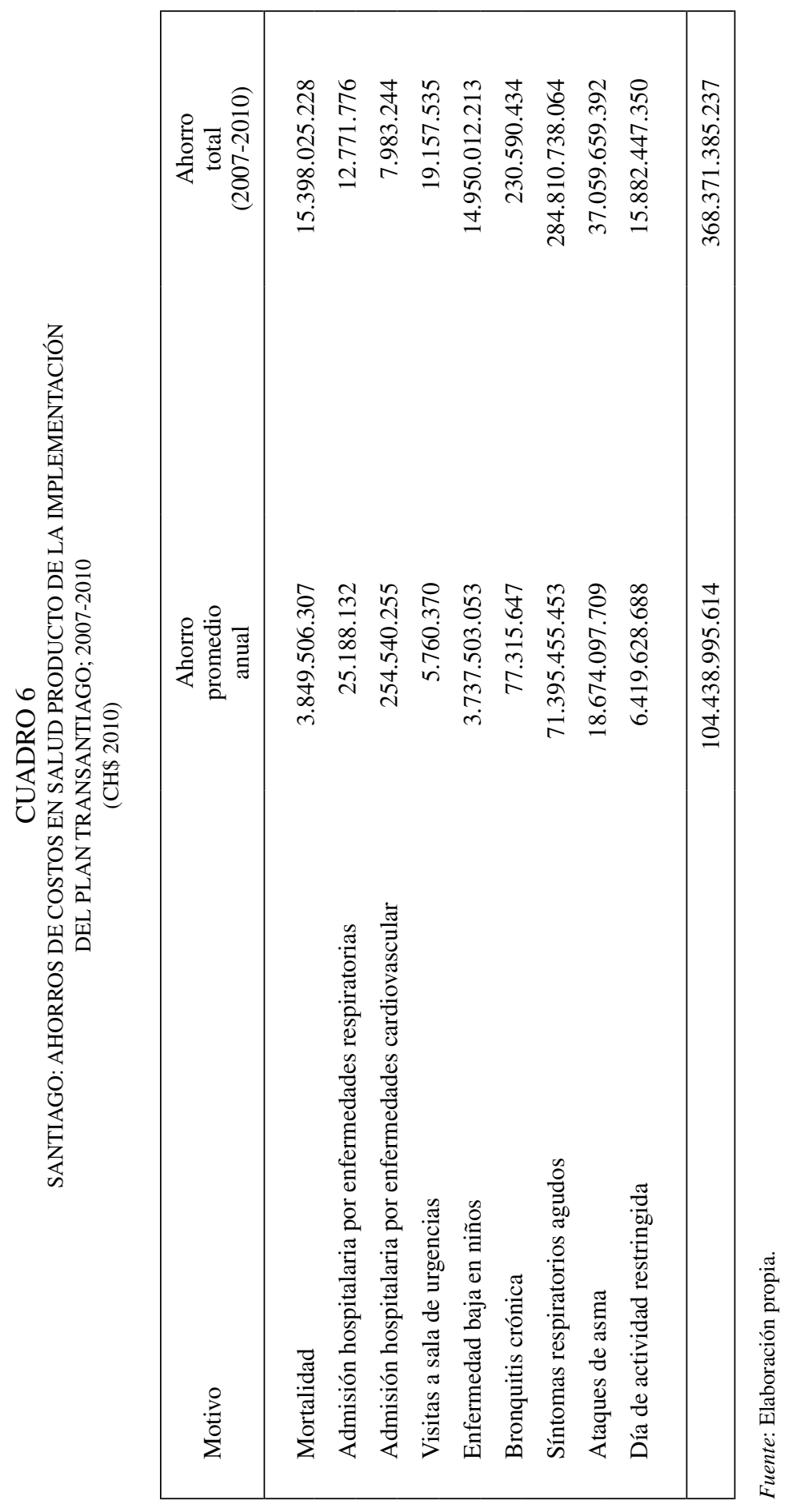




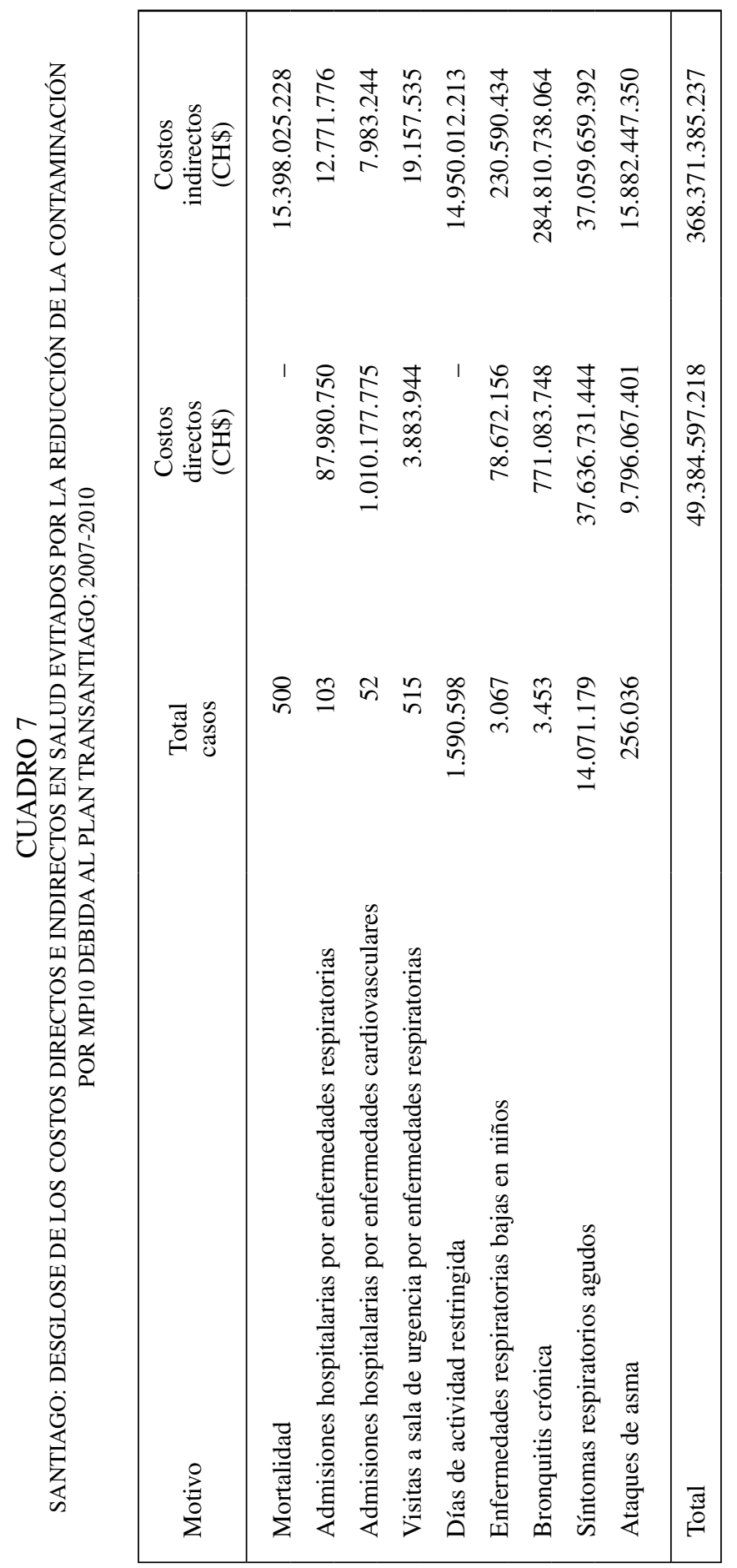


Por último, el Cuadro 7 muestra el desglose de los costos directos e indirectos evitados. Este desglose permite esclarecer qué proporción de este ahorro es percibido por el sector público y cuál por los agentes privados. Se observa que del total de los costos $12,5 \%$ equivale al ahorro fiscal por concepto de menores prestaciones médicas, considerando que este estudio no incluye datos para el sector privado de salud. La mayor parte del ahorro es internalizada por los agentes privados.

\section{Conclusiones}

En el presente trabajo se estimó una disminución de por lo menos $3,9 \mu \mathrm{g} / \mathrm{m}^{3}$ en las concentraciones atmosféricas de MP10 en la ciudad de Santiago desde el año 2007 al 2010. Esto es coincidente con el año de la implementación del plan de reforma y reestructuración del sistema de transporte público de la ciudad, conocido como Transantiago. Las estimaciones del modelo econométrico propuesto y los test de robustez realizados permiten aceptar (no rechazar) la hipótesis planteada de que la reforma del transporte público contribuyó a la descontaminación atmosférica de la capital por MP10. Este efecto se estima aislado de la influencia de otras variables relevantes (confundentes), como las restricciones del suministro de gas proveniente de Argentina, las condiciones meteorológicas, y una tendencia decreciente en el tiempo en los niveles de contaminación en Santiago debido a cambios tecnológicos, de estructura económica, etcétera.

Utilizando una metodología de funciones dosis-respuesta y de valoración económica se obtiene que en promedio cada año, del período 2007-2010, se logra un ahorro de más de CH\$ 104.439 millones (o US\$ 2.216 millones) como consecuencia de la reducción de las concentraciones atribuibles al Plan Transantiago, de los que aproximadamente el $12 \%$ equivale a ahorro fiscal por concepto de gasto en salud.

Cabe señalar además que el ahorro total anual del $\mathrm{CH} \$ 104.439$ por la reducción de la contaminación por MP10 estimada en este trabajo es cercano al subsidio permanente establecido para el plan de transportes de la capital en la Ley $\mathrm{N}^{\circ} 20.378$ del año 2009 y que asciende a $\mathrm{CH} \$ 115.000$ millones anuales.

También es interesante observar que de acuerdo al modelo aquí estimado, los cortes en la provisión de gas natural proveniente de Argentina -que justamente se intensificaron a partir del año 2007- tuvieron un impacto en las concentraciones de material particulado de sentido contrario al provocado por el Transantiago, y que supera en magnitud la reducción estimada como consecuencia de la implementación de dicho plan de modernización del transporte público. En efecto, mientras que el Transantiago redujo la contaminación en cerca de $3,9 \mu \mathrm{g} / \mathrm{m}^{3}$, los días con $100 \%$ de corte de suministro de gas argentino implicaron un aumento de más de $15 \mu \mathrm{g} / \mathrm{m}^{3}$ en las concentraciones diarias de MP10. Estos resultados sugieren que el efecto negativo de estos cortes en el suministro de gas argentino enmascaró el efecto benéfico del Transantiago sobre las concentraciones de MP10, que no se pudo observar inmediatamente después de su puesta en marcha el año 2007.

La bondad de la metodología aquí empleada es que permite aislar el efecto atribuible al Plan Transantiago de los efectos de las variables confundentes 
contemporáneas. Nótese que dicha metodología tiene diversas limitaciones que responden al hecho de trabajar con datos agregados, como también a la escasez de datos relacionados al fenómeno analizado. Para aumentar los niveles de confianza de los beneficios estimados, quedan abiertas algunas líneas de investigación a desarrollar. Primero, la incorporación a los modelos estimados de variables concernientes a la intensidad de uso de los diversos modos de transporte, tanto del parque automotriz como del trasporte público, que permitan esclarecer los cambios en la asignación modal de los viajes. En segundo lugar, avanzar en una identificación geográfica más localizada de los cambios en las concentraciones de contaminantes productos del plan estudiado, lo que permitiría cuantificar de forma más precisa los efectos sobre los segmentos de la población realmente afectados. Por último, es necesario levantar información acabada y actualizada, con base médica, de los costos de los padecimientos de las enfermedades, tanto en su forma directa como indirecta, teniendo en cuenta las variaciones en los precios relativos y las mejoras tecnológicas que se han producido desde el estudio empleado para la valoración (Holz, 2000).

Este trabajo aporta desde una sola dimensión a la valoración completa del plan de modernización del transporte de Santiago, ya que existen otros beneficios tales como posibles reducciones en los tiempos de espera, viajes más expeditos, disminución de la contaminación acústica, reducción de accidentes y mejores condiciones laborales de los choferes, que no fueron contemplados. Debe considerarse también que el análisis realizado representa una mirada parcial del plan, en cuanto no hace referencia a los posibles costos del mismo, como, por ejemplo, el aumento en la evasión del pago por parte de los usuarios, o los efectos del nuevo plan sobre el costo generalizado de viaje de los usuarios.

\section{Bibliografía}

Arellano, M. y S. Bond (1991). "Some tests of Specification for Panel data: Montecarlo Evidence and an Application to Employment Equations", Review of Economic Studies, 58, pp. 277-292.

Atal, R. (2009). "Efectos de las medidas de Gestión de Episodios Críticos en la calidad del aire en Santiago, Chile”, Instituto de Economía, Pontificia Universidad Católica de Chile, Santiago.

Beltrán, P. y C. Palma (2012), “Transantiago, su evolución operacional desde el diseño original". Trabajo presentado en el XVII Congreso Panamericano de Ingenería de Tránsito, Transporte y Logística, 24 al 27 de septiembre, 2012, Universidad de Los Andes.

Centro Mario Molina Chile (2007). Estudio "Evaluación del Impacto de Transantiago en la Calidad del Aire de la Región Metropolitana, año 2007", Informe Final, No BIP 30069882-0.

Clerc, J. (2007). "Valoración de mejoras ambientales en Santiago: Teoría, supuestos y aplicación", Facultad de Ciencias Físicas y Matemáticas, Universidad de Chile, Santiago.

CONAMA Región Metropolitana (2009). "Resultados Plan Operacional para la Gestión de Episodios Críticos de Contaminación Atmosférica por Material Particulado Respirable MP10”. CONAMA RM, Santiago. 
Dictuc (2007), “Actualización del Inventario de Emisiones de Contaminantes Atmosféricos en la Región Metropolitana”, Informe Final, 15 de mayo, Comisión Nacional del Medio Ambiente, Región Metropolitana.

EIU (2010), Índice de Ciudades Verdes en América Latina. Economic Intelligence Unit; publicado por Siemens AG; Munich Alemania, p. 100.

ENAP (2004). Memoria Anual, Santiago.

Fernando, H.J.S., Mammarella, M.C., Grandoni, G., Fedele, P., Di Marco, R., Dimitrova, R. y P. Hyde (2012). "Forecasting PM10 in metropolitan areas: Efficacy of neural networks". Environmental Pollution 163: 62-67.

Ffrench-Davis, R. (2010). Economic Reforms in Chile. From Dictatorship to Democracy, Palgrave Macmillan, Londres.

Gallego, F., J.P. Montero y C. Salas (2011). The Effect of Transport Policies on Car Use: Theory and Evidence from Latin American Cities, Draft, Departmento de Economía, Pontificia Universidad Católica de Chile.

Garreaud, R. D. y J. Rutllant (2006). "Factores meteorológicos de la contaminación atmosférica", en R. Morales (ed.), Contaminación Atmosférica Urbana. Episodios críticos de contaminación ambiental en la ciudad de Santiago, pp. 36-40. Editorial Universitaria. Santiago, Chile.

Gassebner, M., M.J. Lamla y J-E Sturm (2011). Determinants of Pollution: what do we really know?, Oxford Economic Papers, 63, pp. 568-595.

Gómez-Lobo, A. (2012), "The Ups and Downs of a Public Transport Reform: the case of Transantiago", mimeo, Department of Economics, University of Chile.

Holz, J. (2000). "Estimación de Costos Unitarios en Morbilidad y Mortalidad y su aplicación para calcular los beneficios del Plan de Prevención y Descontaminación Atmosférica de la Región Metropolitana”, Facultad de Ciencias Económicas y Administrativas, Universidad de Chile, Santiago.

Judson, R.A. y A.L. Owen (1999). "Estimating dynamic panel data models: a guide for macroeconomists", Economic Letters, 65, pp. 9-15.

Merino, M, (2006). "Factores Episodios de alta concentración de contaminantes: material particulado MP10 y ozono troposférico", en R. Morales (ed.), Contaminación Atmosférica Urbana. Episodios críticos de contaminación ambiental en la ciudad de Santiago, pp. 56-60. Editorial Universitaria. Santiago, Chile.

Merino, M, G. Alvarado, y E. Figueroa (2005). "Aire”, en Geo Chile (ed.), Estado del Medio Ambiente en Chile: Informe País, LOM Impresiones. Santiago, Chile, pp. 37-70.

Ministerio Secretaría General de la Presidencia (1996). Decreto Supremo No 131.

Ostro, B. (1998). "Cómo estimar los efectos de la contaminación atmosférica en Salud", Centro de Estudios Públicos, Santiago.

Sánchez, J., Valdés, S. y B. Ostro (1998). "Estimación de los beneficios en salud del Plan de descontaminación de Santiago", El Trimestre Económico, 363-406.

Sectra (2007). "Análisis Ambiental de las Emisiones del Actual Sistema de Transporte Público de Buses de Santiago año 2007", Secretaría Interministerial de Transportes, Santiago.

Song, S., Wu, Y., Jiang, J., Yang, L., Cheng, Y. y Y. Hao (2012). "Chemical characteristics of size-resolved PM2.5 at a roadside environment in Beijing, China”. Environmental Pollution, 161: 215-221. 
